Revue d'histoire de l'Amérique française

REVUE D.HISTOIRE DE L'AMÉRIQUE FRANÇAISE

\title{
Jean-Baptiste-Nicolas Roch de Ramezay et sa famille, au Canada et en France, après 1760
}

\section{Raymonde Litalien}

Volume 37, numéro 4, mars 1984

URI : https://id.erudit.org/iderudit/304209ar

DOI : https://doi.org/10.7202/304209ar

Aller au sommaire du numéro

Éditeur(s)

Institut d'histoire de l'Amérique française

ISSN

0035-2357 (imprimé)

1492-1383 (numérique)

Découvrir la revue

Citer cette note

Litalien, R. (1984). Jean-Baptiste-Nicolas Roch de Ramezay et sa famille, au Canada et en France, après 1760. Revue d'histoire de l'Amérique française, 37(4), 603-610. https://doi.org/10.7202/304209ar d'utilisation que vous pouvez consulter en ligne.

https://apropos.erudit.org/fr/usagers/politique-dutilisation/ 
NOTE DE RECHERCHE

\section{JEAN-BAPTISTE-NICOLAS ROCH DE RAMEZAY ET SA FAMILLE, AU CANADA ET EN FRANCE, APRES 1760}

RAYMONDE LITALIEN

Archives publiques du Canada

France

Les Ramezay, originaires d'Écosse, se sont installés en France, au $\mathrm{XVI}^{\mathrm{e}}$ siècle, sur de petites seigneuries champenoises et les ont exploitées en pratiquant, à l'occasion, une activité militaire. Ainsi, c'est une fonction d'officier de marine qui a conduit Claude de Ramezay au Canada en 1685 et l'y a maintenu jusqu'à sa mort en 1724. Ses fils se sont orientés dans la même carrière et ses filles ont cherché des alliances matrimoniales avec d'autres familles ${ }^{1}$.

S'ils ont, parfois, pratiqué une activité de bourgeois comme la traite des fourrures ou le commerce du bois, c'était à leur point de vue pour tenir leur rang, remplir leur fonction; ils ne s'assimilaient pas pour autant aux bourgeois mais faisaient toutefois partie de la classe dirigeante, ayant accumulé des biens et constitué un capital au Canada.

Nous chercherons ici à suivre l'histoire de ce capital après 1760 ainsi que celle des Ramezay, aussi bien en France que dans l'ancienne colonie. Pour ce faire, nous nous appuierons principalement sur des documents originaux provenant des descendants de la famille Ramezay, acquis par les Archives du Canada en $1980^{2}$.

Jean-Baptiste-Nicolas Roch de Ramezay, le seul homme de la famille à porter ce nom, n'attend pas la capitulation de Montréal pour rentrer en France: le 20 septembre 1759, il quitte Québec sur un bateau ramenant plus de 700 militaires dans la métropole; le 18 septembre, il avait pris la responsabilité de la reddition de Québec, en tant que «lieutenant pour le Roy» dans cette ville et après une requête des bourgeois. En quittant le sol canadien, Ramezay se sent libéré d'un énorme poids, mais il ne jouira pas longtemps de cette quiétude car sa décision de rendre Québec à l'ennemi a été très fortement contestée de part et d'autre de l'Atlantique et Ramezay doit même se justifier par un mémoire adressé au ministre de la Marine en 1764.

\footnotetext{
$1 \quad$ Voir le tableau généalogique de la famille Ramezay à la fin de l'article.

2 MG 18, H 54, Instruments de recherche $n^{\circ} 1232$.
} 
Dans l'immédiat, même si Ramezay a l'impression d'avoir échappé au désastre, son retour définitif en France est loin d'être glorieux. À 51 ans, après une activité militaire intense et des promotions régulières, il se trouve contraint à une retraite prématurée, avec 800 livres par an de pension annuelle (demi-solde). De plus, il est seul en France pendant 4 ans, alors que son épouse, son unique fille et son gendre sont restés à Québec pour liquider le maximum de leurs biens d'origine «canadienne», c'est-à-dire accumulés au Canada.

C'était d'ailleurs la totalité de leur avoir car il ne restait rien de ce qui leur avait appartenu en France. Quand Claude de Ramezay quitta la Champagne, il partageait avec sa soeur la propriété de quelques parties de fiefs ou de seigneuries, qui leur rapportaient d'ailleurs très peu. Lors de son mariage avec Charlotte Denys de La Ronde, en 1690, il n'avait rien d'autre à apporter que «cinq mille livres de douaire à prendre sur les plus clairs des biens qu'il a de present ou pourra avoir à l'avenir en l'Ancienne France ou en ce pays... ${ }^{3}$ Deux ans après la mort de sa soeur Anne, en 1726, tous les biens de Champagne sont vendus. Les 4500 livres obtenues n'ont servi qu'à payer les dettes de la propriété: entretien, hypothèques des années précédentes, frais encourus par Anne de Ramezay avant sa mort.

Ainsi, à son arrivée en France, Roch de Ramezay ne peut compter ni sur le coin de terre de ses ancêtres, ni sur des revenus provenant de la terre de France. Il arrive avec sa part des biens familiaux ainsi qu'avec ceux qu'il a accumulés lui-même.

\section{Les biens des Ramezay au Canada}

Au cours des 75 ans d'implantation au Canada, la famille Ramezay avait surtout acquis des biens seigneuriaux. Il s'agissait d'un ensemble de fiefs, dont la superficie a varié avec les années, groupés principalement autour de la rivière Richelieu et à l'ouest du lac Champlain, connus au XVIII ${ }^{e}$ siècle sous les noms de seigneuries de Sorel, Chambly, Ramezay, Bourchemin, Monnoir, Yamaska, de même que les fiefs de Saint-Denis, Beauport (ou Poports) et Grande-Anse. Ces propriétés étaient mises à profit par les cultivateurs à qui était octroyée une bande de terrain et qui versaient les redevances au seigneur. Les Ramezay, officiers de marine, n'ont jamais habité sur leurs seigneuries, du moins avant 1760 , et en ont tiré relativement peu de bénéfices. Il serait possible de faire les comptes car les archives Ramezay, récemment acquises, fournissent plusieurs listes de revenus.

Claude de Ramezay, successivement gouverneur de Trois-Rivières et gouverneur de Montréal, recevait, en plus de sa solde, un revenu de

\footnotetext{
3 Québec, 7 novembre 1690, contrat de mariage de Claude de Ramezay, signé par François Genaple, notaire. Archives publiques du Canada, MG 18, H 54. Les autres documents cités dans cet article sont tous conservés aux APC sous cette même cote.
} 
la traite des fourrures, ce qui était interdit aux officiers en fonction, mais largement pratiqué et même toléré par les autorités de la colonie ainsi dispensées d'augmenter les soldes de leurs officiers qui arrivaient à toucher des revenus convenables grâce aux activités de ce commerce. Claude de Ramezay mit aussi sur pied un commerce de bois, à partir de scieries, dans la région de Montréal et celle de la Baie Saint-Paul et, comme il avait obtenu des contrats pour la fourniture de bois aux chantiers de construction navale du Roi, il tira quelque profit de cette activité. Après la mort de son père, Louise reprit ce commerce et le fit prospérer. Si bien qu'à partir de 1739 et pendant plus de 30 ans, elle tirait suffisamment de revenus de cette entreprise et des autres biens de ses parents pour avoir à verser 600 livres de rentes à son frère et à chacune de ses soeurs.

Claude de Ramezay avait aussi acquis des propriétés avec maisons d'habitation, à Québec, rue Sainte-Anne. À Montréal, il en possédait au moins deux dont la demeure de pierre appelée plus tard le Château Ramezay qui, à l'époque de sa construction en 1705, était la plus grande et la plus somptueuse maison particulière de toute la colonie, à la grande satisfaction du gouverneur de Montréal.

En fait, cette belle demeure, contrastant considérablement avec la modestie et même le dénuement des maisons des «habitants», était destinée à une vie mondaine et sociale de grand style. Un exemple type nous en est donné par la fête donnée le 10 décembre 1721 à l'occasion de la signature du contrat de mariage de Louise-Geneviève de Ramezay avec Louis-Henry Deschamps de Boishébert, seigneur de la Bouteillerie et, plus tard, commandant à Détroit. Cette événement réunit la plupart des familles nobles canadiennes: d'Ailleboust, Le Gardeur de Repentigny, La Valtrie, Contrecoeur, Gaultier de Varennes, Robineau de Portneuf, Juchereau du Chesnay, Lacorne de Saint-Luc, Saint-Ours Deschaillons, LeMoyne, Begon, etc.

Claude de Ramezay menait très grand train, rivalisant avec celui du gouverneur Vaudreuil dont il enviait le pouvoir. Il n'empêche qu'après son décès, en 1724, sa famille n'eut plus les moyens de continuer sur le même style et sa veuve chercha à se défaire du «château» dont les simples frais d'entretien étaient trop lourds pour cette femme qui ne touchait que 1000 livres de rente. À cette date, la demeure fut évaluée à 28245 livres par l'intendant Dupuy mais ce n'est qu'en 1745 qu'elle sera vendue à la Compagnie des Indes pour 17600 livres $^{4}$.

Comme bien d'autres Canadiens, les Ramezay subissent une dévaluation de leurs biens alors qu'ils souhaitaient trouver gloire et fortune dans ce pays neuf. Si la propriété foncière, si la «pierre» perdent de

\footnotetext{
réal, 1938).
} 
leur valeur, qu'en sera-t-il du «papier» si largement répandu dans toute la colonie?

Cependant, les enfants Ramezay ne semblent pas s'en inquiéter et vivent largement: des commandes en France de biens de consommation courante montrent bien qu'on ne se vêtait pas seulement «d'étoffe et de laine du pays» et qu'on ne marchait pas souvent en «souliers de beu». En plus de fils, d'aiguilles, de mouchoirs et de toile, on commande des quantités considérables de satin, de mousseline brodée, de rubans, de dentelles, de manteaux élégants, de souliers de velours de damas et de cuir fin, des bijoux et des gants délicats ainsi que de grands vins de Bordeaux. Ces commandes, échelonnées entre 1740 et 1760 , se poursuivent même en période de guerre.

Les Ramezay nés au Canada semblent y avoir vécu dans l'aisance et même le luxe. Roch de Ramezay, qui rentre définitivement en France en 1759, compte sur la vente de ses biens pour s'assurer une retraite confortable, un niveau de vie au moins équivalent à celui qu'il connaissait au Canada.

La maison de la rue Saint-Louis à Québec et la seigneurie de Sorel sont vendues à John Bondfield, en 1764, pour 72000 livres, à répartir entre Ramezay et ses soeurs, c'est-à-dire Geneviève, veuve de Boishébert, Charlotte, supérieure de l'Hôpital-Général de Québec, Louise, célibataire et Élisabeth, épouse de Louis de La Corne, seigneur de Terrebonne. Au moment de la vente, tous les bénéficiaires éprouvent un grand soulagement et sont d'accord pour dire que la vente a été bonne, compte tenu des circonstances - tous les Canadiens qui peuvent partir cherchent à vendre. De plus, les quatre filles Ramezay envient leur frère, sa femme et sa fille de pouvoir quitter le Canada: Louise écrit à Mme de Ramezay, sa belle-soeur: «Je vois que vous prenez le bon parti [d'aller en France]» (10 septembre 1763). Geneviève, qui vit à l'Hôpital-Général de Québec, tient un langage semblable à sa nièce Mme Bellot: «Souvent je voudrais être en France mais on n'entend parler que de vaisseaux perdus, cette année deux, l'année dernière trois cela ne donne point envie de passer... avec les François.» Mme de Ramezay touchera aussi une rente de 7000 livres de la vente d'un des biens (lettre de Thouron, le 23 fé:vrier 1766).

À l'automne 1764, les affaires étant à peu près réglées, Mme de Ramezay rentre en France avec sa fille Charlotte-Marguerite, son gendre Antoine Bellot et leurs enfants. Ils s'installent tous dans la région d'origine de Bellot, seigneur de Segonzac «qui a trouvé son bien très délabré et... y a beaucoup de frais» (lettre à Thouron, 20 avril 1768). Ramezay et son épouse, pour leur part, vivent à Blaye, à proximité de Bordeaux, au nord de l'estuaire de la Gironde. 


\section{Les valeurs des Ramezay en France}

Le soulagement éprouvé au départ du Canada et à la vente des biens a vite laissé place à de nouvelles inquiétudes devant la dévaluation des valeurs mobilières et la difficulté de toucher du numéraire.

Nous connaissons l'essentiel des valeurs du dernier des Ramezay par trois sources principales: les états de comptes établis par Thouron Frères, son chargé d'affaires à La Rochelle, sa correspondance avec le banquier Vincent, rue Vivienne, à Paris, ainsi que la correspondance des époux Ramezay avec leurs parents immédiats restés au Canada.

Le 20 novembre 1764, un premier état de comptes établi par Thouron Frères, de La Rochelle, chiffre les valeurs de Ramezay à 19809 livres $13 \mathrm{~s} .8 \mathrm{~d}$. Cette somme lui rapporte $4 \%$ par an. C'est sur cet intérêt, sur sa pension et éventuellement sur les autres rentes qui lui parviendraient du Canada que Ramezay compte vivre, sans toucher au capital. À cette somme devraient s'ajouter les lettres de change ramenées du Canada par son gendre Antoine Bellot et qui, d'après une liste du 26 juin 1765, se monteraient à 21663 livres.

Un deuxième état de comptes de Thouron Frères, daté du 28 janvier 1770 , chiffre son avoir à 38865 livres. Ses revenus, pendant les cinq années précédentes, s'établissent comme suit:

- l'intérêt de son argent

- l'indemnité britannique, 1 ère et $2^{\mathrm{e}}$ part

- lettres de change, rentes et autres revenus du Canada (estimés, en 1765, à 21663 livres).
4673 livres

1866

14383

Cette somme de 14383 livres comprend toute la liquidation des «papiers du Canada» appartenant à Ramezay. Il ne touchera rien d'autre avant son décès en 1777. À cette date, son solde, chez Thouron, se chiffre encore à 10946 livres.

La complexité de la liquidation du «papier du Canada» est bien connue: entre le 15 octobre 1759 et le 12 mars 1769 , on compte au moins 18 arrêts du Conseil d'État du Roi sur cette question, sans parler des mesures prises par la Grande-Bretagne qui ont su aussi rester dans un flou avantageux. Ramezay s'est trouvé au coeur de ce problème, dans l'incertitude de ses gains jusqu'en 1769. Il s'inquiète particulièrement de ce qu'il arrivera à toucher sur la vente de la seigneurie de Sorel à John Bondfield dont l'argent doit passer par Londres, chez le banquier Vialar, puis chez le banquier Vincent à Paris, puis chez Thouron à La Rochelle. Tous ces hommes d'affaires attendent évidemment d'avoir des certitudes sur la façon de traiter «le papier» avant d'en remettre le produit à leurs clients. 
Ainsi, le 29 novembre 1764, Ramezay apprend par Thouron que la lettre de change de Bondfield est «protestée» car les agents de Paris n'ont pas encore reçu l'argent. Le 23 février 1766, Vialar, à Londres, ne peut payer les lettres de change et autres titres car il est obligé, ainsi que tous les autres Anglais, de faire un serment solennel et authentique que «les papiers qu'ils font liquider appartiennent à des anglais ou à des canadiens sujets de la Grande-Bretagne et non à des français et qu'il ne peut compromettre son honneur, ni sa religion». Pour Ramezay, la liquidation sera accomplie le 3 mai 1768 , avec une perte d'environ 7000 livres.

Entre-temps, en 1766, Ramezay cherche à faire un peu de commerce avec les colonies françaises restantes: des vitres à Saint-Pierre et Miquelon et des clous à Saint-Domingue. Produits qui semblent se vendre difficilement car il attendra 1770 pour toucher $1261.12 \mathrm{~s}$. des vitres de Saint-Pierre et Miquelon et 1773 pour toucher les revenus des clous de Saint-Domingue: 241 livres plus une barrique de café.

Sur le plan proprement financier, Ramezay est gêné par la façon dont se pratiquent les échanges monétaires en France. Il ne peut pas toujours disposer rapidement et à son gré des valeurs en dépôt chez Thouron à La Rochelle: ce dernier n'ayant pas de bureau à Blaye ni à Bordeaux, il fallait donc trouver, chez un ou plusieurs banquiers de Bordeaux, du papier dû à Thouron et pouvant être versé à Ramezay, ce qui prenait parfois quelques semaines. Autrement, Thouron prenait le risque de confier des espèces à un ami ou à un parent qui les remettait en mains propres au propriétaire. Ramezay se lasse de ces procédés et, le 5 avril 1772, retire 10000 livres de chez Thouron pour les déposer chez Latuillière à Bordeaux, espérant faciliter ainsi les opérations.

Le chiffre des dépenses annuelles des Ramezay se monte à 2000 ou 3000 livres par an, provenant des 800 livres de pension, 792 livres d'intérêt chez Thouron, 425 livres d'intérêt chez Vincent, des rentes de Mme de Ramezay et de l'argent pris sur le capital. Les sources susceptibles de révéler la nature de leurs dépenses sont minces mais on n'y trouve aucune trace de frais somptueux; les commandes à Vincent se limitent à une perruque (en 1766) qu'il fera réparer en 1768; à Thouron, il commande un lit de plume, un poêle de faïence, des rognons de castor, des graines de rave et cerfeuil, des plants de raisin. Le 20 avril 1768, Ramezay écrivait à Thouron: «Ma fortune est très médiocre, je ne devois pas m'attendre à finir mes jours dans une aussi grande médiocrité de bien-être. Ce sont des revers de fortune à quoi sont exposés tous les hommes. Si j'avais quelque chose à me reprocher, je serais inconsolable, mais, Dieu merci, je puis aller tête haute partout.» Le 16 juin 1769 , il écrit aussi à son banquier Vincent, à Paris, à qui il ne fait habituellement guère de confidences: «Je ne vous cacherai point que j'ay un très mince revenu.» 
Dans l'ensemble, c'est l'amertume qui marque cette période de la vie de Ramezay, aussi bien envers son pays d'origine qu'envers celui de ses ancêtres. Le 2 mai 1764, il écrit: «La malheureuse affaire de la Bastille $^{5}$ est finie, elle cause bien du chagrin. Pour moi, j'en ai ma bonne part que le bon Dieu soit béni. On y tombe sur les morts et les vivants. Je ne sais quel pays il faut habiter pour n'avoir point de croix, ce n'est pas le Canada, il en fournit qui sont uniques.» Il n'est pas heureux, non plus, dans son nouveau lieu de vie, à Blaye. Dès le 3 février 1765, Thouron lui écrit: «Nous sommes bien fâchés que vous vous ennuyez dans l'endroit où vous êtes.»

Une seule chose semble encore le réjouir, c'est la visite des ressortissants du Canada et les nouvelles de ceux qui y sont restés. Le 18 janvier 1767, il écrit à Thouron, à propos de l'homme d'affaires Charly Saint-Ange: «Nous aurions grand plaisir de le voir pour parler un peu du Canada que je regretterai tant que je vivrai.»

Les Ramezay restent en contact avec beaucoup de Canadiens, surtout des parents directs ou par alliance. On sait qui s'installe en métropole, qui est en voyage et pour combien de temps et l'on essaie de se voir. Les relations sont évidemment plus commodes avec ceux qui vivent en France et il y en a beaucoup parmi les relations des Ramezay: Charly Saint-Ange, Louis Le Gardeur de Repentigny, Raffetot de Boishébert, Douglas de Lisle, Sabrevois, la famille de François-Xavier de SaintOurs, etc.

Avec ceux qui sont restés au Canada, la correspondance s'établit difficilement au début, et même jusque dans les années 1770, il faut trouver des bateaux de Bordeaux ou de La Rochelle pour Londres et, parfois, pour Saint-Pierre-et-Miquelon. Des paquets se perdent mais les relations sont particulièrement suivies avec les Tonnancour. L'image que Ramezay en reçoit est celle d'une famille qui poursuit la carrière militaire avec le gouvernement anglais, qui réussit à obtenir une situation avantageuse dans la nouvelle administration, dont les fils s'illustrent dans la guerre d'indépendance américaine. Le père, Louis-Joseph Godefroy de Tonnancour, qui est colonel des milices et commissaire, touche 4000 livres d'appointements annuels en 1777 et tous jouissent d'un train de vie enviable.

C'est avec cette famille que les Bellot garderont le contact après la mort des Ramezay. Les Tonnancour transmettront ce qui revient à leurs cousins français de la vente de seigneuries aussi bien du côté des Tonnancour (Maskinongé, 1785) que des Ramezay (Chambly, 1784 et Monnoir, 1794). Les Bellot, tous officiers de marine, toucheront leur dernière part de cette vente le 18 février 1796, soit 5 ans après la mort

5 Il s'agit ici du «Procès du Canada» dans lequel ont été impliqués la plupart des fonctionnaires en poste au Canada avant 1760 et, en particulier, l'intendant Bigot. 
de leur grand-mère, Mme de Ramezay. Par la suite, on ne trouve plus trace de relations entre les deux familles.

L'histoire de Jean-Baptiste-Nicolas Roch, le dernier des Ramezay, illustre bien la fuite des capitaux en 1760. Même si cette famille avait cherché à s'enraciner au Canada, les membres qui ont pu en partir se sont empressés de le faire, avec le maximum de leurs biens. Les capitaux restés sur place y ont été laissés contre le gré des possédants.

De plus, la famille Ramezay constitue une manifestation de la chute de la noblesse française, accélérée, avant la Révolution, par son association au destin de la colonie. S'accrochant à la carrière militaire traditionnellement réservée à la noblesse, cette famille a cherché aussi à s'insérer dans le monde des bourgeois en pratiquant le commerce, mais elle n'a pu mener de front ces deux activités appartenant à deux classes sociales encore trop éloignées l'une de l'autre. Le dernier des Ramezay, rejeté de la vie canadienne à cause du rôle joué dans la chute de la colonie, n'a pu réintégrer sa fonction professionnelle en France: le Ministère de la Marine ne lui a pas proposé de poste et l'a mis à la retraite. Après une existence confortable en Nouvelle-France, il a connu toutes les amertumes du déracinement. Ce qu'il ignore, c'est que ses parents, au Canada, vivent le même destin, mais plus étalé dans le temps. Les nobles canadiens, malgré leurs efforts, n'arriveront jamais à s'associer au nouveau pouvoir du vainqueur et à y jouer un rôle important, comme ils l'ont tenté pendant près d'un siècle. Ils verront la chute du régime seigneurial et l'extinction lente de la noblesse canadienne, ce groupe social qui ne trouve plus ni place à prendre ni rôle a jouer. 